\title{
Longitudinal investigation of permeability and distribution of macromolecules in mouse malignant transformation using PET
}

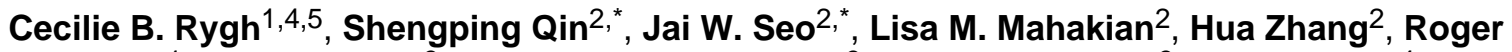 \\ Adamson ${ }^{1}$, Jane Q. Chen ${ }^{3}$, Alexander D. Borowsky ${ }^{3}$, Robert D. Cardiff ${ }^{3}$, Rolf K. Reed ${ }^{4}$, Fitz- \\ Roy E. Curry ${ }^{1,4}$, and Katherine W. Ferrara ${ }^{2}$ \\ ${ }^{1}$ Department of Physiology \& Membrane Biology, University of California. Davis, USA \\ 2 Department of Biomedical Engineering, University of California. Davis, USA \\ ${ }^{3}$ Department of Pathology \& Laboratory Medicine, University of California. Davis, USA \\ 4 Department of Biomedicine, University of Bergen, Norway \\ ${ }^{5}$ Heart and Circulatory Group, Haukeland University Hospital, Bergen, Norway
}

\begin{abstract}
Purpose-We apply positron emission tomography to elucidate changes in nanocarrier extravasation during the transition from premalignant to malignant cancer, providing insight into the use of imaging to characterize early cancerous lesions and the utility of nanoparticles in early disease.
\end{abstract}

Experimental Design-Albumin and liposomes were labeled with ${ }^{64} \mathrm{Cu}$ (half-life 12.7 hours) and longitudinal PET and CT imaging studies were conducted in a mouse model of ductal carcinoma in situ. A pharmacokinetic model was applied to estimate the tumor vascular volume and permeability.

Results-From early time-points characterized by disseminated hyperproliferation, the enhanced vascular permeability facilitated lesion detection. During disease progression, the vascular volume fraction increased 1.6 fold and the apparent vascular permeability to albumin and liposomes increased $\sim 2.5$ fold to 6.6 and $1.3 \times 10^{-8} \mathrm{~cm} / \mathrm{s}$, respectively, with the accumulation of albumin increasing earlier in the disease process. In the malignant tumor, both tracers reached similar mean intratumoral concentrations of $\sim 6 \% \mathrm{ID} / \mathrm{cc}$ but the distribution of liposomes was more heterogeneous, ranging from 1-18\% ID/cc compared with 1-9\% ID/cc for albumin. The tumor-tomuscle ratio was $17.9 \pm 8.1$ and $7.1 \pm 0.5$ for liposomes and albumin, respectively, indicating a more specific delivery of liposomes than with albumin.

Conclusions-PET imaging of radiolabeled particles, validated by confocal imaging and histology, detected the transition from premalignant to malignant lesions and effectively quantified the associated changes in vascular permeability.

\section{Keywords}

albumin; DCIS; liposome; MIN-O; PET

Corresponding author: Professor Katherine W. Ferrara, Department of Biomedical Engineering, UC Davis, Davis, CA 95616.

kwferrara@ucdavis.edu, Phone: (530) 754-9436, Fax: (530) 754-5739.

These authors contributed equally

Disclosure of Potential Conflicts of Interest

No potential conflicts of interest were disclosed. 


\section{Translational relevance}

Detection of cancerous progression is required for differential diagnosis and therapeutic planning. Here, the sensitivity and accuracy of pharmacokinetic modeling using positron emission tomography (PET) are exploited to detect the transition from premalignant to malignant breast cancer lesions through a characterization of the vasculature. The tumor vascular volume fraction is first estimated from carrier-conjugated radioactivity immediately after injection; transport into the interstitium is then estimated. Albumin, a $4 \times 4 \times 14 \mathrm{~nm}$ tracer, has been previously applied to characterize normal vascular function, since a small increase in the number of gaps through or between cells or the formation of vesicular vacuolar organelles significantly increases albumin permeability. We demonstrate that the apparent permeability of ${ }^{64} \mathrm{Cu}$-labeled albumin provides a sensitive indication of tumor transition. ${ }^{64} \mathrm{Cu}$-labeled liposomes augment this measurement by precisely defining the time of transition and by mapping the heterogeneity of permeability.

\section{Introduction}

Sensitive and quantitative measures to characterize cancerous progression are required for differential diagnosis and therapeutic monitoring. Imaging techniques allow us to diagnose and monitor cancer progression longitudinally; positron emission tomography (PET) is a functional imaging technology that provides rapid, reproducible, non-invasive in vivo assessment and quantification of biological processes involved in tumor development. In this study, we detect cancerous progression within a mouse model of ductal carcinoma in situ (DCIS) by using PET to track nanometer-sized tracers. The model recapitulates the clinico-epidemiologic observations in human disease and is referred to as mouse mammary intraepithelial neoplasia outgrowths (MIN-Os), which consistently undergo malignant transformation to a phenotype capable of autonomous ectopic growth (1-3). The MIN-O model allows us to study the pharmacokinetics and tumoral uptake of PET-labeled albumin and liposomes at various points in the developmental timeline of mammary tumors (4). Our aim was to determine the utility of PET to detect the tumor transition through a detection of changes in vascular volume fraction and permeability. While 2- $\left[{ }^{18} \mathrm{~F}\right]-$ fluoro-deoxy-Dglucose $\left({ }^{18} \mathrm{~F}-\mathrm{FDG}\right)$ has previously been applied to follow changes in tumor metabolism (5), PET-based imaging of nano-structures such as liposomes has not previously been applied to follow the malignant transformation, and ${ }^{18} \mathrm{~F}-\mathrm{FDG}$ was unable to detect the vascular changes associated with the malignant transition (5).

In order to accurately assess the timing of the transition, we combine measurements obtained with radiolabeled albumin and liposomes. Albumin ( $\sim 66 \mathrm{kDa}$, dimensions of $4 \times 4 \times 14 \mathrm{~nm})$ has been extensively used for permeability studies $(6,7)$, and is a versatile protein carrier for targeting of hydrophobic molecules in clinical settings. The preferential localization of albumin in tumors and inflamed tissue due to increased vascular permeability, availability, biodegradability as well as lack of toxicity and immunogenicity also make it a good candidate for drug delivery (8). Liposomes are vesicles composed of one or more concentric phospholipid bi-layers and are considered to be a prototype of all nanocarriers under development (9). The typical diameter of liposomes is $65-120 \mathrm{~nm}$, with the small diameter facilitating extravasation through leaky blood vessels and fusion with cellular membranes $(10,11)$. Prolonged blood circulation of the liposomes is achieved with the addition of a polyethylene glycol (PEG) coating to resist opsonization, which efficiently minimizes their removal by macrophages $(12,13)$. Liposomes facilitate controlled release of drugs, thereby reducing drug-related toxicity and facilitating targeted delivery of drugs. 
On the way to their target, tracers or drug carriers meet physiological barriers, such as interstitial hypertension and abnormal tumor vasculature (14-16). The altered tumor endothelium includes modified intercellular junctions as well as specialized transendothelial pathways such as vesicular vacuolar organelles (VVOs), fenestrations and transendothelial gaps, often characterized as an effective "pore size cutoff" (17-21). Elevated levels of permeability enhancing factors, including bradykinin, nitric oxide (NO) and vascular endothelial growth factor (VEGF) create these changes and can be therapeutic targets for neutralization (22-25). As a result of the altered endothelial morphology, nanoparticles of the optimal size exhibit enhanced permeability and retention (EPR) within the tumor interstitium (24). Permeability is particularly enhanced for small carriers (i.e. $<10$ $\mathrm{nm}$ ) but interstitial retention is more pronounced for larger particles (16). Reduced lymphatic drainage further increases the retention of molecules in the tumor interstitium. Diffusion of larger particles is less than of smaller particles, slowing the redistribution of large particles to the blood circulation. Thus, larger tracers are required to detect and map vascular changes associated with the tumor transition.

In order to quantify such changes, we evaluate a PET-based pharmacokinetic model (26) and augment PET with confocal imaging. Many non-invasive techniques have been developed to evaluate particle pharmacokinetics (27-40). While the spatial resolution of MRI (magnetic resonance imaging) and CT (computed tomography) is superior to PET, PET has advantages in sensitivity and quantitation, since picomolar concentrations can be detected and estimates of blood pool and organ tracer concentration accurately mapped in time and space. The 12.7 hour half-life of ${ }^{64} \mathrm{Cu}$ facilitates intra-tumoral particle tracking over 48 hours; repeated dosing facilitates imaging of longitudinal disease progression over months of disease progression. Thus, we apply ${ }^{64} \mathrm{Cu}$-labeled albumin and liposomes to track intra-tumoral delivery over the progression from the premalignant to malignant phenotype and evaluate the ability of PET to characterize disease progression.

\section{Materials and Methods}

Details of each procedure are described in the supplementary methods.

\section{Tumor model}

MIN-O is a mouse model of DCIS that recapitulates "premalignant" disease of the mammary gland. The course of disease approximates the neoplastic progression of human breast carcinoma from pre-invasive DCIS to invasive carcinoma. Outgrowths transplanted into gland-cleared mammary fat pads of syngeneic immunocompetent mice result in hyperplastic and dysplastic growth, which remains confined to the dimensions of the stroma and is analogous to human DCIS (3).

\section{Mice}

All animal studies were conducted under a protocol approved by the University of California, Davis Animal Use and Care Committee.

\section{Experimental protocol}

Three studies were conducted ( $\mathrm{n}=27$ total), involving MIN-O subline 4 (tumor latency of 5 weeks) in the first two studies and subline D (latency of 7-8 weeks) in the third study. The first group of animals (line 4, $\mathrm{n}=8$ ) was PET and CT scanned at 3, 5, 7 and 8 weeks after transplantation to follow the development of tumors longitudinally. A second cohort was implanted with the same tumor line $(\mathrm{n}=8)$ and sacrificed after PET scanning at 5 weeks in order to catch the transformation from premalignant to malignant tissue. A third cohort of 
animals (line $\mathrm{D}, \mathrm{n}=11$ ) was imaged with confocal microscopy and PET at 5 and 7 weeks post-transplantation.

Tracers

Tumor permeability and distribution of PEGylated liposomes and albumin were investigated. Liposomes were prepared as described previously $(26,40,41)$ and had an average diameter of 100-115 $\mathrm{nm}$. Fatty acid and globumin-free bovine serum albumin (BSA, A0281) was purchased from Sigma-Aldrich (St. Louis, MO, 99\%, MW of $67 \mathrm{kDa}$ ).

\section{PET imaging}

In vivo and ex vivo imaging was performed with a microPET scanner (Focus 120, Siemens Medical Solutions, Inc). Animals that received radiolabeled liposomes were imaged at 0, 6, 18, 28 and 48 hours after tail-vein injection and data were acquired for $30 \mathrm{~min}$. Due to the shorter half-life of albumin, animals receiving ${ }^{64} \mathrm{Cu}$-BSA were scanned at $0-2,6$ and 18 hours after injection (up to 48 hours for cohort 2), where data were acquired for 2 hours and re-binned into a dynamic scan for the $0-2$ hour time-point.

\section{Confocal imaging}

To investigate the co-localization of vessels and tracers, animals were injected through the tail vein with Alexa-555 conjugated tracers and lectin for vessel identification (Fluorescein Lycopersicon esculentum (tomato) lectin from Vector laboratories, CA ( $2 \mathrm{mg} / \mathrm{ml}, 50 \mu \mathrm{l})$ ).

\section{Image analysis}

The tumor tracer concentration, transport constant, $\lambda\left(\mathrm{h}^{-1}\right)$, vascular volume fraction $(\%)$, and apparent permeability (AP, $\mathrm{cm} / \mathrm{s}$ ) were estimated based on the time activity curves (TACs) for each week and for each tumor using an image-driven pharmacological model developed by Qin et al, 2009 (26).

\section{Results}

After implantation of the hyperplastic lesions, thin layers of hyper-proliferative cells expanded through the mammary fat pad, inducing substantial angiogenesis prior to tumor formation (Supplementary Figure 1). At week 3, even small non-palpable lesions were detected by PET with both albumin and liposomal tracers ( 8 out of 16 lesions were palpable but all were detected by PET). At five weeks after implantation, solid tumors were observed at scattered foci, with a fibrous stroma replacing the fat pad in the tumor vicinity (Fig. 1A and B). Regions with high intensity on ex vivo PET images corresponded geometrically with regions of dense outgrowth or tumor tissue (Fig. 1A and B) and were also readily visualized with in vivo imaging (Fig. 1C). Even at this early time-point after transplantation, the accumulation for both albumin and liposomes was greater in the lesion than in the healthy fat pads and surrounding muscle, where the accumulation of the tracers did not exceed the background level (Fig. 1C). At 5 weeks after implantation and 48 hours after injection, the average tumor accumulation across the two tracers was $\sim 4.3 \% \mathrm{ID} / \mathrm{g}$ (with albumin accumulation exceeding liposomal accumulation) and was significantly greater than the accumulation of $0.6 \% \mathrm{ID} / \mathrm{g}$ in surrounding striated muscle tissue ( $\mathrm{p}<0.05$, ANOVA). Muscle and tumor accumulation was similar between PET and biodistribution data (Fig. 1D), and $e x$ vivo and in vivo PET estimates of radioactivity in identical breast tumors and fat pads were correlated $\left(\mathrm{R}^{2}=0.95\right.$, least square analysis).

In vivo PET imaging was then applied to visualize and quantify vascular density and permeability longitudinally over tumor development (Fig. 2). Serial imaging of a group of animals was conducted as the tumors developed from the premalignant to malignant state 
(Fig. 2A) over weeks 3, 5, 7 and 8 after implantation, alternating the injection of liposomal (Fig. 2A panels ii and iv) and albumin (panels i and iii) tracers. In the same animal, as the tumors progressed, the accumulation of both tracers increased as detected by increased PET signal intensity in the lesions (Fig. 2A).

Image-based estimates of the tumor volume increased rapidly starting around 5 weeks after transplantation (Fig. 2B), indicating a transition from a premalignant to a malignant phenotype at this time-point. The tumor doubling time decreased as the tumors progressed; the mean tumor doubling time pre-transition ( $3-5$ weeks), at the transition (5-7 weeks) and post-transition (7-8 weeks) was $13.3 \pm 6.8,11.4 \pm 6.4$ and $8.4 \pm 4.5$ days, respectively $(\mathrm{p}=0.13$, ANOVA between pre- and post-transition) (Fig. 2C). The mean tumor doubling time (in days) for the entire time period was $9.5 \pm 2.8$ days. PET images of the liposomal (Fig. 2D upper) and albumin (Fig. 2D lower) tracer distribution correlated with histology, where regions of necrosis were visible. Cystic regions were prominent in the majority of the tumors at the 8-week time-point. The tumor mass detected on CT images corresponded with regions of increased intensity on PET images for both tracers (Fig. 2D). By the 8-week timepoint, regions of dense tumor tissue were observed with extensive vasculature (Fig. 2D).

\section{Albumin uptake precedes liposomal uptake in tumor transition}

Lesion growth and progression were reflected in the tumoral tracer uptake, which increased as the transition occurred (Fig. 3). Liposomes had a longer half-life in blood than albumin (i.e. $\sim 18$ and $\sim 6$ hours, respectively) thus enabling accumulation of the larger liposomal tracer over a longer period of time (Fig. 3A); in each case scanning continued until the blood concentration was less than $10 \% \mathrm{ID} / \mathrm{cc}$. After removing the estimated blood pool concentration, the tumor accumulation of liposomes demonstrated a step-wise transition between the premalignant (weeks 3-5) and malignant (weeks 7-8) state, with the temporal peak accumulation increasing up to three fold between weeks 3-5 and 7-8 (Fig. 3B).

The transition in the accumulation of the much smaller albumin molecule was more gradual, where differences in peak accumulation were significant between weeks 3-5, 3-7, 3-8 and $7-8$ (Fig. 3C, p<0.05, ANOVA). Comparing the time activity curves of the two tracers, the time to peak accumulation was shorter for albumin; in week 8 , accumulation within the first hour was as high as 3\% ID/cc (Fig. 3B-C) and accumulation of 3\% ID/cc required 6 hours for liposomes. The rate of clearance of the tracers over 48 hours was similar. At week 5 after implantation, $25.5 \pm 16.9 \%$ of the liposomal tracer had cleared from tumors by 48 hours, as compared with $33.6 \pm 9.9 \%$ of the albumin tracer (data not shown) ( $\mathrm{p}=0.06$, ANOVA).

The tumor concentration of the tracer at the time of the latest scan (i.e. 48 hours for liposome injection and 18 hours for albumin injection) reached $\sim 6 \% \mathrm{ID} / \mathrm{cc}$ at week 8 and was confirmed by the biodistribution (Fig. 3B-D). The tumor-to-muscle ratio was significantly higher with the liposomal tracer, i.e. $17.9 \pm 8.1$ and $7.1 \pm 0.5$ for liposomes and albumin respectively, indicating a more specific delivery of liposomes than with albumin. In general, liposomal radioactivity and the radioactive metabolites were concentrated in the spleen and intestines, while radioactivity was greater in liver, kidney and muscle tissues after the injection of ${ }^{64} \mathrm{Cu}$-labeled albumin. The accumulation in blood, heart, and lungs was similar between the two tracers.

\section{PET facilitates direct visualization of tumor heterogeneity}

Imaging the same animal at weeks 3 and 7 or 5 and 8 , mean accumulation in the same tumors increased 1.6 and 2.3 fold, respectively, for the albumin and liposomal tracers ( $<<0.02$, paired t-test) (Fig. 4A). The initial accumulation in week 3 and final accumulation in week 8 was similar for the two tracers. Thus, for liposomes, accumulation increased more 
rapidly at the time of the transition (between weeks 5 and 7) whereas albumin accumulation gradually increased between weeks 3, 5, 7 and 8 (Fig. 4B).

In order to assess tumor heterogeneity, estimates of maximum and minimum accumulation were generated with a resolution of $(0.8 \mathrm{~mm})^{3}$ and compared with the mean over the tumor (Fig. 4B). Comparing liposomal and albumin accumulation, the spatial maximum and the heterogeneity of liposomal accumulation were larger (Fig. 4), where the spatial variation of liposome accumulation ranged from $<1$ to $\sim 18 \% \mathrm{ID} / \mathrm{cc}$ and the spatial variation of albumin accumulation was smaller, ranging from $\sim 1$ to $9 \% \mathrm{ID} / \mathrm{cc}$. At 8 weeks after implantation, the accumulation of albumin and liposomes (peak over time and average over animals) was 5.7 \pm 0.5 and $6.0 \pm 1.1 \% \mathrm{ID} / \mathrm{cc}$, respectively, reflecting the similar mean and greater heterogeneity of liposomal accumulation. While the minimum tumor accumulation observed following liposome injection was smaller than accumulation following albumin injection, the difference was not significant.

PET images also demonstrated the differences in the rate of accumulation and intra-tumoral heterogeneity of the two tracers (Fig. 4C-D). Images and surface plots of a single slice through the tumor center at the 18-hour time-point 8 weeks after implantation convey spatial differences in tumoral tracer distribution between the two tracers. Tumors displayed a heterogeneous pattern of tracer distribution (Fig. 4C), with a minimum in the tumor center in which activity accumulated slowly. The high intra-tumoral heterogeneity of the liposomal tracer was reflected by a larger spatial variance in intensity, with high accumulation peripherally and large central sub-regions with lower accumulation. The rapid accumulation of the albumin tracer was also clearly observed (Fig. 4D i and iii), indicating a more rapid extravasation of the smaller-sized tracer into the tumor interstitium. Across the tumor, the spatial variation in albumin accumulation was less than that observed for liposomes (Fig. 4D iv). For example, the mean accumulation of albumin and liposomes in these central slices was $5.1 \pm 1.5$ and $5.4 \pm 3.9 \% \mathrm{ID} / \mathrm{cc}$, respectively.

\section{Vascular volume fraction and permeability increase with tumor transition}

The tumor vascular volume fraction (estimated from tumor radioactivity immediately after injection) increased from $5 \%$ to nearly $8 \%$ during the progression from the premalignant to malignant phenotype (Fig. 5A) and was similar for the liposomal and albumin tracers. CD31 images confirmed an extensive vasculature and the development of large supplying arteries in weeks 7 and 8 (Fig. 2A). The vascular volume fraction was also estimated based on the CD31-positive endothelium and increased from $\sim 3$ to $\sim 8 \%$ over the transition (Fig. $5 \mathrm{~B}$ ), with an increase in median vessel diameter from 10 to $15 \mu \mathrm{m}$, respectively. Segmenting out sub-regions within the tumor revealed intra-tumoral heterogeneous perfusion, with vascular volume sub-fractions reaching $10 \%$ and $16 \%$ in highly vascularized areas in albumin and liposomes tumors, respectively (data not shown).

The apparent vascular permeability increased approximately two-fold during the transition from the premalignant to malignant phenotype, peaking near $1.3 \times 10^{-8} \mathrm{~cm} / \mathrm{s}$ for liposomes and $6.6 \times 10^{-8} \mathrm{~cm} / \mathrm{s}$ for albumin (Fig. $5 \mathrm{C}$ ). The change in AP to albumin was gradual, with an increase of $\sim 1 \times 10^{-8} \mathrm{~cm} / \mathrm{s}$ between weeks 3 to 5 and 7 to 8 and a larger increase of $2.8 \times$ $10^{-8} \mathrm{~cm} / \mathrm{s}$ between weeks 5 and 7 (Fig. 5D). Permeability to liposomes increased in a stepwise fashion, with a substantial change observed only between weeks 5 and 7 (Fig. 5D). There was also large intra-tumor heterogeneity in permeability, where the variation in AP ranged between 2-3.8 fold for albumin and 1.2-1.6 fold for liposomes. 


\section{Confocal imaging of tracers validates kinetics and accumulation at the time of transition}

In order to visualize the microscopic distribution of the tracers in ex vivo tumor interstitium, fluorescent albumin and liposomes were imaged together with a vascular lectin after circulation for 0-28 hours. Immediately after injection of the labeled liposomes (i.e. 30 minutes), minimal, isolated fluorescence was observed; however, at 18 and 28 hours, particle fluorescence had increased with co-localization of vessel walls (in green) and liposomes (Fig. 6A). With fluorescent albumin, immediately after injection, the tracer was concentrated near the tumor vasculature, whereas at 18 hours, the tracer distribution was homogenous within the tumor interstitium (Fig. 6B). By 28 hours, the majority of the remaining tracer was detected near the tumor vessels. The dynamics of the tracers are visualized in Fig. 6C and D, where the measurements within the ROIs placed adjacent to vessels differed for the two tracers, indicating that the albumin distribution within the tumors is dynamic, clearing more rapidly than the liposomes. With both tracers, particle fluorescence was much lower in healthy muscle than in the tumors (Fig. 6A and B).

\section{Discussion}

Quantitative measurement of biochemical processes underlies progress in cancer biology and treatment. Here, we demonstrate that in vivo PET imaging can detect the transformation from a premalignant to malignant breast cancer by quantifying the extravasated tumor concentration of tracers and the vascular volume fraction and blood level of tracer at each time-point. Small $\left(\sim \mathrm{mm}^{3}\right)$ lesions were detected by microPET using ${ }^{64} \mathrm{Cu}$-based tracers and correlated with foci of early disease. The spatial resolution of the microPET scanner used in this study was $<2 \mathrm{~mm}$ and was sufficient to demonstrate to detect and map accumulation in this model; similar resolution is possible in future human studies as well. While other modalities have advantages in spatial and temporal resolution, the sensitivity of PET to picomolar concentrations of material and its inherent quantitation of pharmacokinetic parameters suggest that PET is an obvious choice for such studies. PET estimates of changes in both vascular volume fraction and permeability were straight forward and robust; such estimates of vascular and extravascular kinetics are problematic with other imaging methods.

Leveraging the understanding of albumin exchange in normal and other hyperpermeable vascular beds, we employ an albumin tracer to detect early changes in permeability in the MIN-O model of ductal carcinoma in situ. The MIN-O lines provide insight into the origin, evolution and outcome of disease $(1,2,5,42)$. Accumulation of the tracers was minimal in normal fat pads and surrounding striated muscle, validating that the enhanced uptake in the transplanted MIN-Os was due to progressing disease. We found that the vascular volume fraction increased from 3 to $8 \%$ and the apparent vascular permeability to albumin and liposomes increased $\sim 2$-fold to 6.6 and $1.3 \times 10^{-8} \mathrm{~cm} / \mathrm{s}$, respectively, over the transition.

Accumulation of the $4 \times 4 \times 14 \mathrm{~nm}$ albumin tracer increased earlier in the disease progression than the 100-nm liposome tracer and was more gradual, although ultimately the accumulation was similar in the fully malignant lesions (Fig. 4B). An increase in accumulation can be accounted for in terms of increased convection and diffusion through modified intercellular junctions as well as specialized trans-endothelial pathways such as VVOs and trans-endothelial gaps. These mechanisms can contribute to both albumin and liposome transport but their relative contributions in different tumors are controversial (20). Although our measurements of AP do not discriminate between these mechanisms, we emphasize the effectiveness of the albumin tracer to detect early changes in permeability. Specifically, albumin normally crosses the vascular permeability barrier via restricted penetration through "small pores" whose size is determined by junctional proteins and matrix structures within the intercellular junctions (21). Albumin, with an AP of $1 \cdot 10^{-8} \mathrm{~cm} / \mathrm{s}$ in normal muscle, represents the upper limit of normal permeability and increases as a result 
of an increased number of larger pores such as those associated with gaps through or between adjacent cells or the formation of VVOs. These changes can be detected with a tracer the size of albumin because a small increase in the number of such large pores significantly increases albumin permeability. An increase in the accumulation of lower molecular weight tracers can result from an increase in the number of vessels, an increase in the number of small pores or a transition to larger pores; smaller tracers are therefore both less specific and less sensitive to changes in the number of large pores. Although the small molecule tracer ${ }^{18} \mathrm{~F}$-FDG detected the progressive increase in tumor metabolism in a previous study (5), changes in vascular volume fraction and permeability were detected only with the larger probes employed here. Furthermore, albumin is still small enough to easily penetrate pores that may restrict tracers as large as liposomes.

The early increase in albumin accumulation (Fig. 3C, week 3-5 and Fig. 5D) before liposomal accumulation increased (Fig. 3B, week 5-7 and Fig. 5D) and the subsequent increase in permeability to both tracers is consistent with the formation and increase in frequency of a large pore pathway; however, the contribution of specific mechanisms involving VVOs, transcellular gaps or the redistribution of proteins controlling intercellular junction permeability cannot be determined (20). Similarly, the initial transition is indicated by the increase in albumin permeability without as large a change in liposomal permeability.

The above argument emphasizes the effectiveness of albumin (or a tracer with similar size and properties) as a tracer to follow tumor progression. Additional factors influence the net delivery of tracers of different size. The blood half-life of the albumin and liposomal tracers applied here were 6 and 18 hours, respectively, and the improved permeability of the albumin was balanced by the loss in circulation time. The tumor-to-muscle ratio was significantly improved with liposomes as compared with albumin, limiting off-target effects.

Further, a comparison of the accumulation of the two tracers emphasized the heterogeneity in the effective permeability size cutoff. By week 8 , both tracers reached similar mean intratumoral concentrations of $\sim 6 \% \mathrm{ID} / \mathrm{cc}$ (Fig. $4 \mathrm{~B}$ ) but the distribution of liposomes was more heterogeneous reaching concentrations as high as 18\% ID/cc (as compared to a 9\% ID/ cc peak concentration of albumin) (Fig. 4B). High IFP and low diffusion and convection will influence the penetration depths of liposomes, as reported in multicellular tumor spheroids $(43,44)$ and in experimental solid tumors $(45)$ and thus the relative distribution of the tracers is likely to indicate the distribution of large pores. PET is an effective method to map the relative concentration of tracers over time and space, where maximum-intensityprojection images and contour maps were each useful in visualizing variations in accumulation. Due to this tumoral heterogeneity, fully treating a large tumor with a 100-nm particle may be difficult as a larger dose is required to reach a minimum concentration throughout the tumor. Thus, treatment of in situ disease could be facilitated by the development of long-circulating carriers with a diameter on the order of tens of nanometers. There is a substantial literature detailing differences in the accumulation of radiolabeled antibodies (46); such studies will be important for new nanotechnologies as well.

In summary, we find that a $4 \times 4 \times 14 \mathrm{~nm}$ radiotracer provided a sensitive probe to detect the progression from a premalignant to malignant lesion and facilitated a quantitative estimate of vascular volume fraction and apparent vascular permeability. In addition, mapping enhanced permeability to a 100-nm liposomal tracer characterized the timing of the transition while also mapping the spatial heterogeneity of the size-dependent permeability. PET, as employed here, can quantitatively assess tumor progression and inform choices in drug vehicles. 


\section{Supplementary Material}

Refer to Web version on PubMed Central for supplementary material.

\section{Acknowledgments}

We acknowledge the following assistance: Katie Bell (immunohistochemistry), Dave Kukis (radiolabeling of albumin), Jennifer Fung and Douglas Rowland (imaging), Jinxiu Cheng-Liao (image reconstruction) and Mario Hlawitschka (image segmentation).

Grant Support

NIHR01CA103828, NIHR01CA134659 and the Western Norway Regional Health Authority (Helse Vest).

\section{References}

1. Damonte P, Hodgson JG, Chen JQ, Young LJ, Cardiff RD, Borowsky AD. Mammary carcinoma behavior is programmed in the precancer stem cell. Breast Cancer Res. 2008; 10:R50. [PubMed: 18522749]

2. Maglione JE, McGoldrick ET, Young LJ, Namba R, Gregg JP, Liu L, et al. Polyomavirus middle Tinduced mammary intraepithelial neoplasia outgrowths: single origin, divergent evolution, and multiple outcomes. Mol Cancer Ther. 2004; 3:941-53. [PubMed: 15299077]

3. Maglione JE, Moghanaki D, Young LJ, Manner CK, Ellies LG, Joseph SO, et al. Transgenic Polyoma middle-T mice model premalignant mammary disease. Cancer Res. 2001; 61:8298-305. [PubMed: 11719463]

4. Abbey, Craig K.; Borowsky, Alexander D.; Gregg, Jeffery P.; Cardiff, Robert D.; Cherry, Simon R. Preclinical Imaging of Mammary Intraepithelial Neoplasia with Positron Emission Tomography. J Mammary Gland Biol Neoplasia. 2006; 11:137-149. [PubMed: 17091397]

5. Abbey CK, Borowsky AD, McGoldrick ET, Gregg JP, Maglione JE, Cardiff RD, et al. In vivo positron-emission tomography imaging of progression and transformation in a mouse model of mammary neoplasia. Proceedings of the National Academy of Sciences of the United States of America. 2004; 101:11438-43. [PubMed: 15277673]

6. Adamson RH, Lenz JF, Zhang X, Adamson GN, Weinbaum S, Curry FE. Oncotic pressures opposing filtration across non-fenestrated rat microvessels. J Physiol. 2004; 557:889-907. [PubMed: 15073281]

7. Curry FR, Rygh CB, Karlsen T, Wiig H, Adamson RH, Clark JF, et al. Atrial natriuretic peptide modulation of albumin clearance and contrast agent permeability in mouse skeletal muscle and skin: role in regulation of plasma volume. J Physiol. 2010; 588:325-39. [PubMed: 19948658]

8. Kratz F. Albumin as a drug carrier: design of prodrugs, drug conjugates and nanoparticles. J Control Release. 2008; 132:171-83. [PubMed: 18582981]

9. D’Souza GG, Weissig V. Subcellular targeting: a new frontier for drug-loaded pharmaceutical nanocarriers and the concept of the magic bullet. Expert Opin Drug Deliv. 2009; 6:1135-48. [PubMed: 19708822]

10. Drummond DC, Noble CO, Hayes ME, Park JW, Kirpotin DB. Pharmacokinetics and in vivo drug release rates in liposomal nanocarrier development. J Pharm Sci. 2008; 97:4696-740. [PubMed: 18351638]

11. Ferrara KW, Borden MA, Zhang H. Lipid-shelled vehicles: engineering for ultrasound molecular imaging and drug delivery. Acc Chem Res. 2009; 42:881-92. [PubMed: 19552457]

12. Allen TM, Austin GA, Chonn A, Lin L, Lee KC. Uptake of liposomes by cultured mouse bone marrow macrophages: influence of liposome composition and size. Biochim Biophys Acta. 1991; 1061:56-64. [PubMed: 1995057]

13. Klibanov AL, Maruyama K, Torchilin VP, Huang L. Amphipathic polyethyleneglycols effectively prolong the circulation time of liposomes. FEBS Lett. 1990; 268:235-7. [PubMed: 2384160]

14. Jain RK. Tumor physiology and antibody delivery. Front Radiat Ther Oncol. 1990; 24:32-46. discussion 64-8. [PubMed: 2187763] 
15. Jang SH, Wientjes MG, Lu D, Au JL. Drug delivery and transport to solid tumors. Pharm Res. 2003; 20:1337-50. [PubMed: 14567626]

16. Torchilin VP, Lukyanov AN. Peptide and protein drug delivery to and into tumors: challenges and solutions. Drug Discov Today. 2003; 8:259-66. [PubMed: 12623240]

17. Dvorak AM, Feng D. The vesiculo-vacuolar organelle (VVO): A new endothelial cell permeability organelle. Journal of Histochemistry \& Cytochemistry. 2001; 49:419-31. [PubMed: 11259444]

18. Kohn S, Nagy JA, Dvorak HF, Dvorak AM. Pathways of macromolecular tracer transport across venules and small veins - structural basis for the hyperpermeability of tumor blood vessels. Laboratory Investigation. 1992; 67:596-607. [PubMed: 1279271]

19. Nagy JA, Benjamin L, Zeng HY, Dvorak AM, Dvorak HF. Vascular permeability, vascular hyperpermeability and angiogenesis. Angiogenesis. 2008; 11:109-19. [PubMed: 18293091]

20. Bates DO. Vascular endothelial growth factors and vascular permeability. Cardiovascular Research. 2010; 87:262-71. [PubMed: 20400620]

21. Michel CC, Curry FE. Microvascular permeability. Physiol Rev. 1999; 79:703-61. [PubMed: 10390517]

22. Dreher MR, Liu W, Michelich CR, Dewhirst MW, Yuan F, Chilkoti A. Tumor vascular permeability, accumulation, and penetration of macromolecular drug carriers. J Natl Cancer Inst. 2006; 98:335-44. [PubMed: 16507830]

23. Dvorak HF, Sioussat TM, Brown LF, Berse B, Nagy JA, Sotrel A, et al. Distribution of vascular permeability factor (vascular endothelial growth factor) in tumors: concentration in tumor blood vessels. J Exp Med. 1991; 174:1275-8. [PubMed: 1940805]

24. Maeda H, Wu J, Sawa T, Matsumura Y, Hori K. Tumor vascular permeability and the EPR effect in macromolecular therapeutics: a review. J Control Release. 2000; 65:271-84. [PubMed: 10699287]

25. Yuan F, Chen Y, Dellian M, Safabakhsh N, Ferrara N, Jain RK. Time-dependent vascular regression and permeability changes in established human tumor xenografts induced by an antivascular endothelial growth factor/vascular permeability factor antibody. Proceedings of the National Academy of Sciences of the United States of America. 1996; 93:14765-70. [PubMed: 8962129]

26. Qin S, Seo JW, Zhang H, Qi J, Curry FR, Ferrara KW. An imaging-driven model for liposomal stability and circulation. Mol Pharm. 2010; 7:12-21. [PubMed: 19621944]

27. Ponce AM, Viglianti BL, Yu DH, Yarmolenko PS, Michelich CR, Woo J, et al. Magnetic resonance imaging of temperature-sensitive liposome release: Drug dose painting and antitumor effects. Journal of the National Cancer Institute. 2007; 99:53-63. [PubMed: 17202113]

28. Hamoudeh M, Kamleh MA, Diab R, Fessi H. Radionuclides delivery systems for nuclear imaging and radiotherapy of cancer. Adv Drug Deliv Rev. 2008; 60:1329-46. [PubMed: 18562040]

29. Oku N. Delivery of contrast agents for positron emission tomography imaging by liposomes. Advanced Drug Delivery Reviews. 1999; 37:53-61. [PubMed: 10837726]

30. Phillips WT, Goins BA, Bao A. Radioactive liposomes. Wiley Interdisciplinary Reviews: Nanomedicine and Nanobiotechnology. 2009; 1:69-83. [PubMed: 20049780]

31. Ahkong QF, Tilcock C. Attachment of ${ }^{99} \mathrm{~m}$ Tc to lipid vesicles containing the lipophilic chelate dipalmitoylphosphatidylethanolamine-DTTA. Int J Rad Appl Instrum B. 1992; 19:831-40. [PubMed: 1428912]

32. Phillips WT, Rudolph AS, Goins B, Timmons JH, Klipper R, Blumhardt R. A simple method for producing a technetium-99m-labeled liposome which is stable in vivo. Int J Rad Appl Instrum B. 1992; 19:539-47. [PubMed: 1399684]

33. Gabizon A, Papahadjopoulos D. Liposome formulations with prolonged circulation time in blood and enhanced uptake by tumors. Proc Natl Acad Sci U S A. 1988; 85:6949-53. [PubMed: 3413128]

34. Harrington KJ, Mohammadtaghi S, Uster PS, Glass D, Peters AM, Vile RG, et al. Effective targeting of solid tumors in patients with locally advanced cancers by radiolabeled pegylated liposomes. Clin Cancer Res. 2001; 7:243-54. [PubMed: 11234875]

35. Hwang KJ, Merriam JE, Beaumier PL, Luk KF. Encapsulation, with high efficiency, of radioactive metal ions in liposomes. Biochim Biophys Acta. 1982; 716:101-9. [PubMed: 6807354] 
36. Marik J, Tartis MS, Zhang H, Fung JY, Kheirolomoom A, Sutcliffe JL, et al. Long-circulating liposomes radiolabeled with $\left[{ }^{18}\right.$ F $]$ fluorodipalmitin ([ ${ }^{18}$ F $]$ FDP). Nucl Med Biol. 2007; 34:165-71. [PubMed: 17307124]

37. Urakami T, Akai S, Katayama Y, Harada N, Tsukada H, Oku N. Novel amphiphilic probes for $\left[{ }^{18} \mathrm{~F}\right]$-radiolabeling preformed liposomes and determination of liposomal trafficking by positron emission tomography. J Med Chem. 2007; 50:6454-7. [PubMed: 18052025]

38. Mougin-Degraef M, Jestin E, Bruel D, Remaud-Le Saec P, Morandeau L, Faivre-Chauvet A, et al. High-activity radio-iodine labeling of conventional and stealth liposomes. J Liposome Res. 2006; 16:91-102. [PubMed: 16556552]

39. Awasthi V, Yee SH, Jerabek P, Goins B, Phillips WT. Cerebral oxygen delivery by liposomeencapsulated hemoglobin: a positron-emission tomographic evaluation in a rat model of hemorrhagic shock. J Appl Physiol. 2007; 103:28-38. [PubMed: 17615284]

40. Seo JW, Zhang H, Kukis DL, Meares CF, Ferrara KW. A novel method to label preformed liposomes with $64 \mathrm{Cu}$ for positron emission tomography (PET) imaging. Bioconjugate Chem. 2008; 19:2577-84.

41. Paoli EE, Kruse DE, Seo JW, Zhang H, Kheirolomoom A, Watson KD, et al. An optical and microPET assessment of thermally-sensitive liposome biodistribution in the Met-1 tumor model: Importance of formulation. J Control Release. 2010; 143:13-22. [PubMed: 20006659]

42. Namba R, Maglione JE, Young LJT, Borowsky AD, Cardiff RD, MacLeod CL, et al. Molecular Characterization of the Transition to Malignancy in a Genetically Engineered Mouse-Based Model of Ductal Carcinoma In situ. Molecular Cancer Research. 2004; 2:453-63. [PubMed: 15328372]

43. Kostarelos KED, Papakostas A, Yang WH, Ballangrud A, Sgouros G. Binding and interstitial penetration of liposomes within avascular tumor spheroids. Int J Cancer. 2004; 112:713-21. [PubMed: 15382056]

44. Kostarelos KED, Papakostas A, Yang WH, Ballangrud AM, Sgouros G. Engineering lipid vesicles of enhanced intratumoral transport capabilities: correlating liposome characteristics with penetration into human prostate tumor spheroids. J Liposome Research. 2005; 15:15-27. [PubMed: 16194925]

45. Eikenes L, Tari M, Tufto I, Bruland OS, de Lange Davies C. Hyaluronidase induces a transcapillary pressure gradient and improves the distribution and uptake of liposomal doxorubicin (Caelyx) in human osteosarcoma xenografts. Br J Cancer. 2005; 93:81-8. [PubMed: 15942637]

46. Sedlacek HH, Seeman G, Hoffman D, Czech, Lorenz P, Kolar C, Bossler K. Antibodies as carriers of cytotoxicity. Contributions to Oncology. 1992; 43:74-5. 


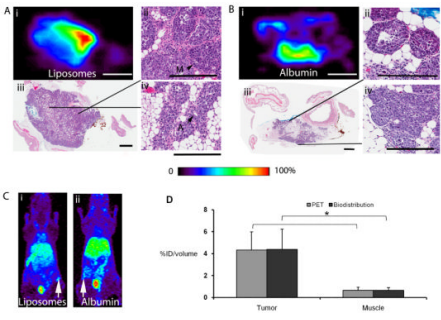

Fig. 1.

PET and histological images at the time of transition. Ai) Ex vivo PET image acquired 48 hours after injection of Cu-labeled liposomes at week 5 (scalebar $5 \mathrm{~mm}$ ). Aii) H\&E showing increased mitotic cells $(\mathrm{M})$, indicating an area of transformation to carcinoma. This area corresponds geometrically with the region in Ai) with high tracer uptake $(20 \times$, scalebar 200 $\mu \mathrm{m})$. Aiii) Low-power H\&E of the MIN-O precancer filling the precleared fat pad with early carcinoma of the same tumor (scalebar $1.5 \mathrm{~mm}$ ) as in Ai). Regions with increased liposome uptake in $\mathrm{Ai}$ ) correspond geometrically with regions in the H\&E section with high cellular density and less residual fat. Aiv) High-power H\&E of acinar and ductal structures with high intra-lesional heterogeneity ( $20 \times$, scalebar $200 \mu \mathrm{m}$, A-acini). Bi) Ex vivo PET image at 48 hours after injection of Cu-labeled albumin is homogenous with lower uptake than observed with liposomes in Ai) (identical image settings) (scalebar $5 \mathrm{~mm}$ ). Bii) Precancerous region with well differentiated cells and ducts $(20 \times$, scalebar $200 \mu \mathrm{m})$. Biii) Low-power H\&E of the same lesion as in $\mathrm{Bi}$ ) with the MIN-O precancer filling the precleared fat pad (scalebar $1.5 \mathrm{~mm}$ ). Biv) The edge of the precancerous region at higher power (20x, scalebar $200 \mu \mathrm{m})$. Ci and ii) Maximum-intensity-projection (MIP) images at 48 hours of the same mice as in $\mathrm{Ai}$ ) and $\mathrm{Bi}$ ) respectively. D) PET measurements in tumor and striated muscle correspond well with biodistribution data. White arrows indicate tumors. PET image color scale from 0 $100 \%$. 

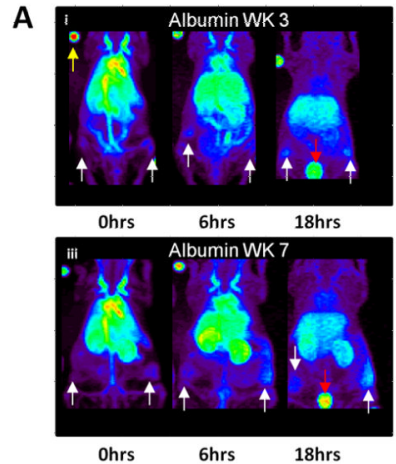

Ohrs

C

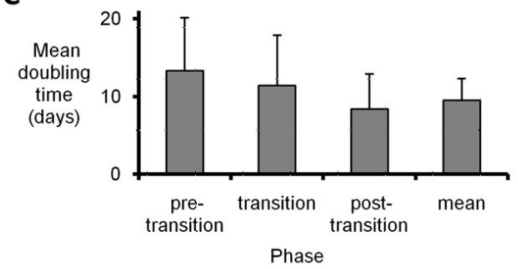

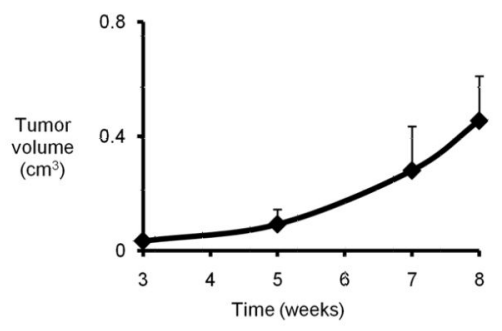

iposomes WK 8

D
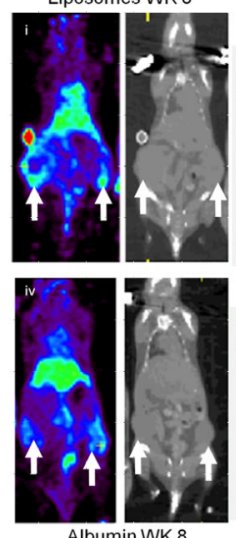
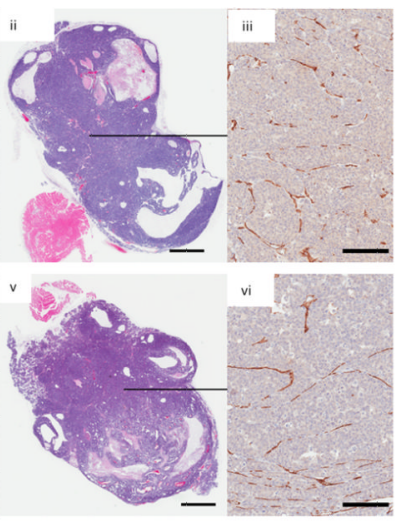

Fig. 2.

Longitudinal development and malignant transformation. MIP images of a single animal as the tumors progressed at Ai) 3, Aii) 5, Aiii) 7 and Aiv) 8 weeks after transplantation of premalignant tissue from a donor. Filled white arrows indicate tumors, red arrows bladder and yellow arrows fiducial markers. B). A rapid increase in tumor growth was observed after week 5, reflecting a transition from premalignant to malignant. C) Tumor doubling time (in days) in the pre-transition phase (3-5 weeks), transition (5-7 weeks), post-transition phase (7-8 weeks). D) Images and histology post-liposome (i-iii) and -albumin (iv-vi) injection at 8 weeks after implantation. i, iv) Co-registered PET and CT images of an animal 18 hours post-liposome (i) or -albumin (iv) injection. ii, v) H\&E histomorphology of tumors in $\mathbf{i}$, iv demonstrate densely packed tumor cells and large cystic regions (scalebar $1.5 \mathrm{~mm}$ ), iii, vi) High-power CD31 section of the tumor center demonstrates large collapsed vessels $(10 \times$, scalebar $150 \mu \mathrm{m})$. PET image color scale from 0 to $100 \%$ as in A. 


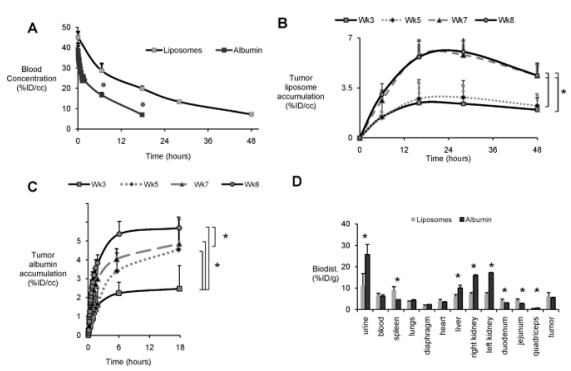

Fig. 3.

Tracer kinetics and biodistribution. A) Circulation of PET-labeled liposomes and albumin averaged over all weeks and animals. B) Tumoral liposome accumulation (average over animals after blood radioactivity is subtracted) vs time after injection as a function of weeks after implantation. Transition to a malignant phenotype between 5 and 7 weeks increases accumulation in step-wise fashion. C) Tumoral albumin accumulation (average over animals after blood radioactivity is subtracted) vs time after injection as a function of weeks after implantation. Transition to a malignant phenotype between 5 and 7 weeks gradually increases accumulation. D) Biodistribution after injection of albumin and liposomes in week 8. *P-value $<0.05$ (image data analyzed with ANOVA and biodistribution data with MannWhitney test) 


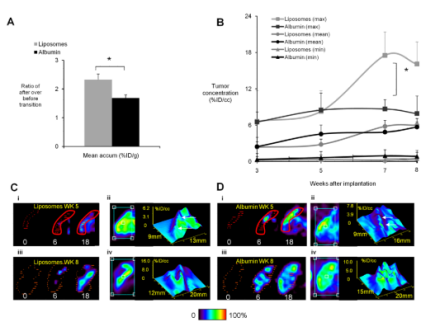

Fig. 4.

Tumor accumulation and heterogeneity. A) Ratio of whole tumor mean tracer accumulation (at peak over time) after vs before tumor transition for the same animals and both tracers. B) Spatial mean, minimum and maximum of tumor concentration after subtracting the radioactivity in the tumor vasculature (peak over time and averaged over animals) versus weeks after implantation for albumin and liposomal tracers. Mean uptake of both tracers increased with disease progression. Maximum tumor uptake of the liposomal tracer is higher after the transition from the premalignant to malignant phenotype than with the albumin tracer reflecting greater heterogeneity. Spatial minimum accumulation of both tracers is < $1 \%$ ID/cc. C) -D) Tumor PET accumulation images and surface plots of liposomes (C) and albumin (D) at 5 weeks (upper row (i-ii)) and 8 weeks (lower row (iii-iv)). In i) and iii) the color map is described in the methods section. In ii) and iv) color map is scaled to 0-100\% for each tumor. Both before and after the transition the albumin tracer accumulates faster than the larger-sized liposomal tracer, visualized by a higher intensity at 6 and 18 hours. Liposomal accumulation is heterogeneous with minimum in tumor center at week 8. Orange/ red lines delineate the tumor tissue, and z-axes are in $\% \mathrm{ID} / \mathrm{cc}$. ${ }^{*} \mathrm{P}$-value $<0.05$ in $\mathrm{A}$ ) paired t-test, in B) ANOVA). 

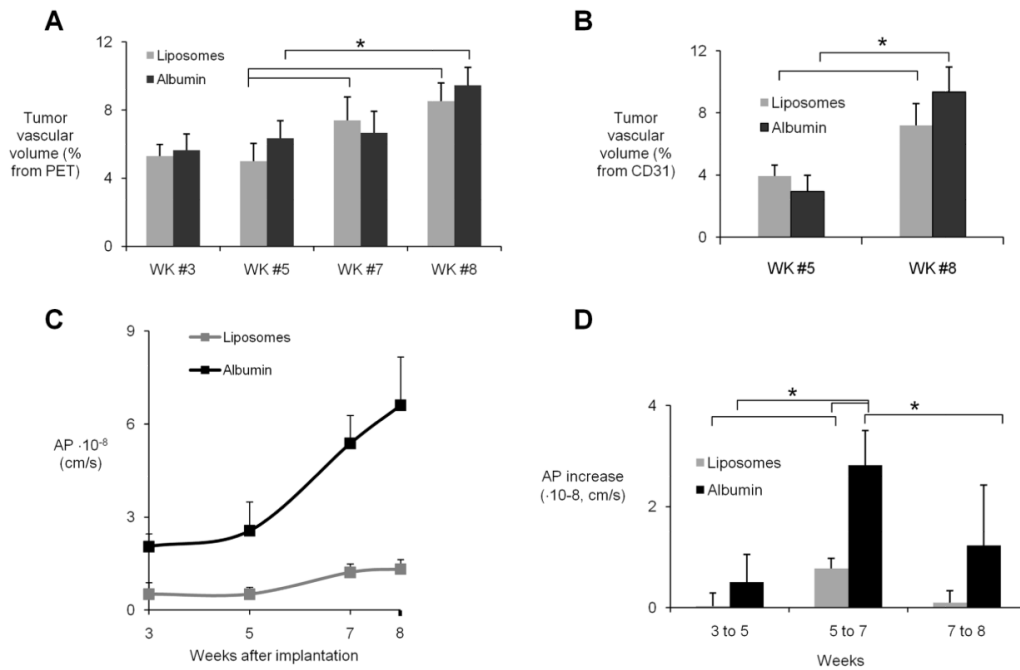

D

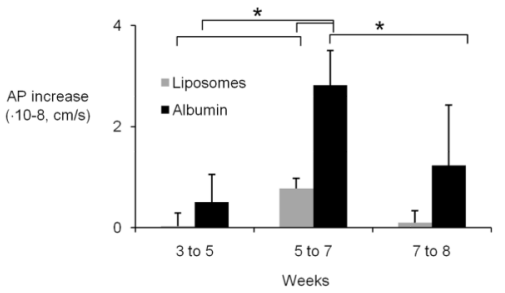

Fig. 5.

Vascular volume fraction and permeability. A) Estimated vascular volume fraction based on PET images versus weeks after implantation. B) Vascular volume fraction estimated by CD31-stained sections versus weeks after implantation. C) Mean tumor apparent permeability (AP) of both tracers increases with increasing tumor volume. AP of the albumin tracer is significantly higher than liposomes at all points $(\mathrm{p}<0.05)$. D) Change in AP over time for each tracer. ${ }^{*} \mathrm{p}<0.05$ tracers at the same time-point (ANOVA). 


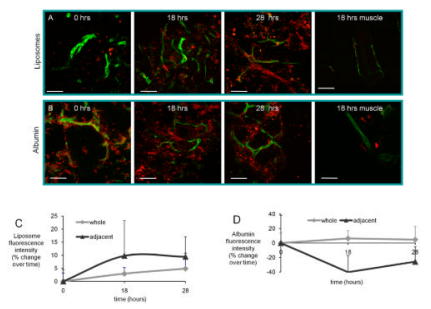

Fig. 6.

Tracer kinetics and accumulation on a microscopic level. A) Confocal images of tumor tissue captured at 0,18 and 28 hours (3D) after injection of Alexa-555-liposomes and lectin and at 18 hours in striated muscle. B) Corresponding images after injection of Alexa-555albumin and lectin in tumor tissue and in striated muscle (3D of $0 \mathrm{hr}$ tumor). C) The kinetics of the labeled liposomes in different regions within the tumor, where the interstitium shows increasing fluorescence intensity over time compared to the 0-hour time-point, indicating retention of the tracer. D) The kinetics of the Alexa-555-labeled albumin, which accumulates and clears more rapidly than in $\mathbf{C}$ ), indicating an enhanced permeability but lower retention of the albumin. Scalebar all images $50 \mu \mathrm{m}$. 